\title{
Impact of neighbourhood selection methods on mobile LiDAR data classification using machine learning algorithms
}

\author{
Salem Morsy $^{\mathrm{a}, *}$, Mahmoud Mohamed $^{\mathrm{b}}$, Adel El-Shazly ${ }^{\mathrm{a}}$ \\ ${ }^{a}$ Public Works Department, Faculty of Engineering, Cairo University, Giza, Egypt, (smorsy, adel.shazly)@eng.cu.edu.eg \\ ${ }^{b}$ Civil Engineering Department, Faculty of Engineering, Fayoum University, Fayoum, Egypt, maa58@fayoum.edu.eg \\ * Corresponding author
}

Keywords: Neighbourhood selection, LiDAR, Classification, Machine Learning

\begin{abstract}
:
Road features classification is essential for different applications such as autonomous vehicles road management. For such objective, mobile laser scanning (MLS) systems provide dense point clouds covering most of road features. Although MLS systems provide sufficient points for each road feature, the main challenge is to find sufficient number of neighbourhood points for each LiDAR point, and hence assign each LiDAR point to its correct class. In this context, we evaluate three neighbourhood selection methods, namely K-nearest neighbour (KNN), spherical and cylindrical along with using machine learning (ML) algorithms for classification step.
\end{abstract}

Our methodology started with defining the three neighbourhood selection methods. The KNN neighbourhood selection method assigns a fixed number of $\mathrm{k}$ nearest points to a query point $\mathrm{x}$ of LiDAR data using the Euclidean distance. Spherical neighbourhood selection method searches all points within a sphere with a predefined radius $r$ of a query point $\mathrm{x}$, while cylindrical neighbourhood selection method searches all points within in 2D projection with a predefined radius $r$ of a query point $x$. Second, fifteen point features, divided into three categories: covariance, moment (Hackel et al., 2016) and height (Weinmann et al., 2017), were then extracted as provided in Table 1. The first seven covariance features were defined using the normalized Eigenvalues (Weinmann et al., 2015), while the Sum feature was derived using the Eigenvalues (Mohamed et al., 2021a). Finally, three ML algorithms, namely Random Forest (RF), Gaussian Naïve Bayes (GNB), and Quadratic Discriminant Analysis (QDA) were carried out (Mohamed et al., 2021b).

\begin{tabular}{|c|c|c|}
\hline \multirow{9}{*}{ Covariance } & $\mathrm{L}_{\lambda}:$ Linearity & $\frac{\mathrm{e}_{1}-\mathrm{e}_{2}}{\mathrm{e}_{1}}$ \\
\hline & $\mathrm{P}_{\lambda}$ : Planarity & $\frac{\mathrm{e}_{2}-\mathrm{e}_{3}}{\mathrm{e}_{1}}$ \\
\hline & $\mathrm{S}_{\lambda}:$ Scattering & $\frac{e_{3}}{e_{1}}$ \\
\hline & $\mathrm{O}_{\lambda}:$ Omni variance & $\sqrt[3]{\mathrm{e}_{1} \mathrm{e}_{2} \mathrm{e}_{3}}$ \\
\hline & $\mathrm{A}_{\lambda}:$ Anisotropy & $\frac{\mathrm{e}_{1}-\mathrm{e}_{3}}{\mathrm{e}_{1}}$ \\
\hline & $E_{\lambda}$ : Eigen entropy & $\sum_{i=1} e_{i} \ln \left(e_{i}\right)$ \\
\hline & $C_{\lambda}$ : Change of curvature & $e_{3}$ \\
\hline & $\sum_{\lambda}:$ Sum & $\lambda_{1}+\lambda_{2}+\lambda_{3}$ \\
\hline & V: Verticality & $-\left\langle(0,0,1), v_{3}\right\rangle$ \\
\hline \multirow{2}{*}{ Moment } & $1^{\text {st }}$ order, $1^{\text {st }}$ axis & $\left\langle\left(p_{i}-p\right), v_{1}\right\rangle$ \\
\hline & $1^{\text {st }}$ order, $2^{\text {nd }}$ axis & $\left\langle\left(p_{i}-p\right), v_{2}\right\rangle$ \\
\hline
\end{tabular}




\begin{tabular}{|c|l|c|}
\hline & $2^{\text {nd }}$ order, $1^{\text {st }}$ axis & $\sum_{\mathrm{i} \in \mathrm{p}}\left\langle\left(\mathrm{p}_{\mathrm{i}}-\mathrm{p}\right), \mathrm{v}_{1}\right\rangle^{2}$ \\
\cline { 2 - 3 } & $2^{\text {nd }}$ order, $2^{\text {nd }}$ axis & $\sum_{\mathrm{i} \in \mathrm{p}}\left\langle\left(\mathrm{p}_{\mathrm{i}}-\mathrm{p}\right), \mathrm{v}_{2}\right\rangle^{2}$ \\
\hline \multirow{2}{*}{ Height } & $\Delta z$ & $\mathrm{Z}_{\max }-\mathrm{Z}_{\min }$ \\
\cline { 2 - 3 } & $\sigma z$ & Standard deviation of $\mathrm{z}$ coordinate within the neighbourhood \\
\hline
\end{tabular}

Table 1. LiDAR point features.

The study area of this research is a road located in Lille, France. A part of Paris-Lille-3D benchmark, which is available at http://npm3d.fr/paris-lille-3d, was used to evaluate the three neighbourhood selection methods. Lille part is about1.5 $\mathrm{km}$ long road with more than 98 million points. The dataset is classified into ground, building, pole, bollard, trashcan, barrier, pedestrian, car and vegetation. Figure 1 shows the overall accuracy of the tested cases. Cylindrical neighbourhood selection method provides the highest overall accuracy of the three methods by $92.4 \%, 78.5 \%$, and $78.2 \%$ for RF, GNB, and QDA, respectively.

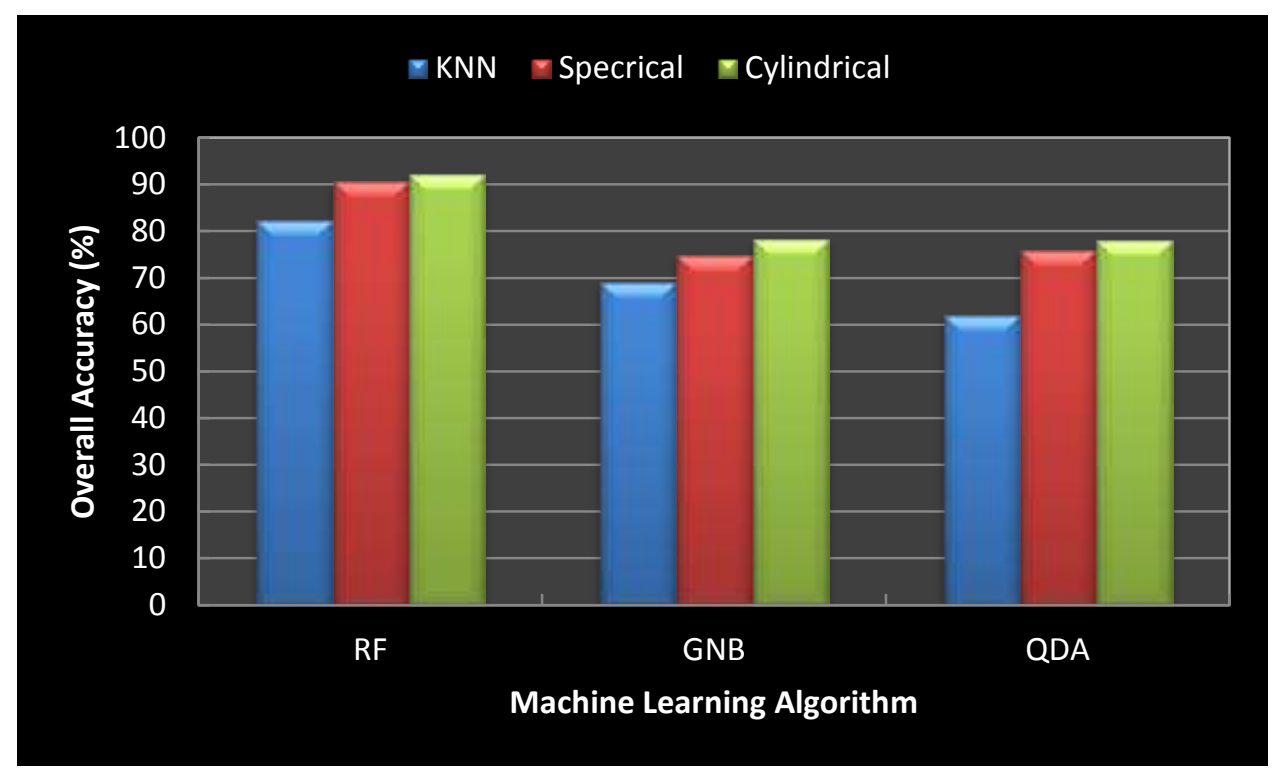

Figure 1. Overall classification accuracy.

\section{References}

Hackel, T., Wegner, J.D. and Schindler, K., 2016. Fast semantic segmentation of 3D point clouds with strongly varying density. Int. Arch. Photogramm. Remote Sens. Spatial Inf. Sci., 3, pp.177-184. https://doi.org/10.5194/isprsannals-iii3-177-2016.

Mohamed, M., Morsy, S. and El-Shazly, A., 2021. Evaluation of data subsampling and neighbourhood selection for mobile LiDAR data classification. The Egyptian Journal of Remote Sensing and Space Science. https://doi.org/10.1016/j.ejrs.2021.04.003.

Mohamed, M., Morsy, S., and El-Shazly, A., 2021. Machine learning for mobile LiDAR data classification of 3D road environment, Int. Arch. Photogramm. Remote Sens. Spatial Inf. Sci., XLIV-M-3-2021, PP.113-117. https://doi.org/10.5194/isprs-archives-XLIV-M-3-2021-113-2021.

Weinmann, M., Jutzi, B., Hinz, S. and Mallet, C., 2015. Semantic point cloud interpretation based on optimal neighborhoods, relevant features and efficient classifiers. ISPRS Journal of Photogrammetry and Remote Sensing, 105, pp.286-304. https://doi.org/10.1016/j.isprsjprs.2015.01.016.

Weinmann, M., Jutzi, B., Mallet, C., Weinmann, M., 2017. Geometric features and their relevance for 3D point cloud classification. Int. Arch. Photogramm. Remote Sens. Spatial Inf. Sci., 4 (1W1), PP.157-164. https://doi.org/10.5194/isprs-annals-IV-1-W1-157-2017. 